\title{
THE ANALYTICAL DESCRIPTION OF REGULAR LDPC CODES CORRECTING ABILITY
}

\author{
Leonid Uryvsky ${ }^{1}$, Serhii Osypchuk ${ }^{2}$ \\ Institute of Telecommunication Systems \\ National Technical University of Ukraine "Kyiv Polytechnic Institute" \\ Kyiv, Industrialny Street, 2, Ukraine, 03057 \\ E-mails: ${ }^{1}$ leonid_uic@ukr.net; ${ }^{2}$ serhii.osypchuk@ieee.org
}

\begin{abstract}
The analytical description of regular LDPC (Low-Density Parity Check) codes correcting ability has been investigated. The statistical dependencies for the maximum number of corrected bits per the code word as a function of LDPC code word length and code rate are given based on multiple experimental analyses of LDPC check matrices. The analytical expressions are proposed for the cases of linear, exponential and polynomial approximations of given results. The most exact analytical formula is proved by criterion of the minimum divergence between the experimental and theoretical results.
\end{abstract}

Keywords: LDPC code, correcting ability, analytical description

\section{Introduction}

The main task of telecommunication (TC) is the information transmission from a source (S) to a recipient $(\mathrm{R})$. Withal it is implied that there is significant distance between $\mathrm{S}$ and $\mathrm{R}$, and this means a necessity of message transformation to the appropriate form for transmission through a communication channel. As known, communication channel can be built based on copper wire, optical fibre, metal waveguide and wireless environment.

One of the most widely used environments for signal transmission in TC systems is a wireless environment. It is used for communication for both very big distances (satellite, tropospheric, radio relay communication) and small distances (mobile cellular communication, wireless local networks). At the same time, the quality criterion for wireless digital TC systems is the bit error probability (BER) of received bits $p_{\text {bit }}$ on the receiver side, i.e. information reliability. The requirements for BER can stand in limits $10^{-5} \ldots 10^{-9}$. Real BER value in practice depends on a number of TC system parameters and parameters of propagation medium.

One of the methods to increase the reliability for information transmission is an anti noise coding that allows improving the information transmission reliability at the expense of energy, frequency and time resources of a communication channel. This article is devoted to one of anti noise coding methods, namely LDPC (Low-Density Parity Check), which is one of the most effective anti noise codes on a number of parameters among other known anti noise coding methods.

LDPC codes are linear block codes with cyclic decoding procedures (Gallager, 1963). Due to specific coding and decoding procedures of LDPC codes, the exact analytical model does not exist for determination of their correcting ability $t$, bits, as function of code word length $n$, bits, and code rate, $R$. As known, code rate $R$ is the ratio of information bits number $k$ in a code word to a total number of all the bits $n$ in a code word: information $k$ and redundant $r=n-k$. Other known anti noise codes such as BCH (Bose-Chaudhuri-Hocquenguem) codes have the exact analytical model for determination their correcting ability $t$ based on other known code parameters $n, R$ (Uryvsky, Prokopenko and Pieshkin, 2011). Thus, the task exists for determination the correcting ability of $t$, bits as function of code word length $n$ and code rate $R$ based on the analysis of multiple experiments on the LDPC code check matrices and detection their properties. It's expected that obtained dependency $t=f(n, R)$ allows us to indicate the number of corrected bits per one LDPC code word without searching the check matrix $H$ with some best parameters. Many works in the LDPC codes area (MacKay and Neal, 1996; Luby, Mitzenmacher, Shokrollahi and Spielman, 1998; Ping and Phamdo, 1999; Honary, Kou and Lin, 2004; Ohtsuki, 2007) are devoted to a study of LDPC codes correcting ability, but an attempt to generalize results into the analytical expression is not made. The results presented in this article are development of work (Osypchuk, 2014) and are devoted to the search of analytical description for regular LDPC codes correcting abilities: $t=f(n, R)$. 


\section{Task Statement}

Generation of regular LDPC codes is defined by consecutive procedure.

Regular LDPC code with code word length $n$ is formed based on check matrix $H$, which is characterized by constant number of one's $W_{\mathrm{r}}$ in every line and constant number of one's $W_{\mathrm{c}}$ in every column (Gallager, 1963). Check matrix $H$ has low density of one's (the density of one's is considered as low if relative part of one's is less than $50 \%$ of all elements (ones and zeros) in check matrix).

Correcting ability $t$ of LDPC code depends on check matrix parameters $n, W_{\mathrm{r}}, W_{\mathrm{c}}$. The position of ones in check matrix $H$ is formed based on casual columns permutations in basic submatrix that contains only single one in each column. Thus, a regular LDPC code rate $R$ is determined by a formula:

$R=\frac{n-\left(n \cdot \frac{W_{c}}{W_{r}}-\left(W_{c}-1\right)\right)}{n}=1-\frac{W_{c}}{W_{r}}+\frac{W_{c}-1}{n}$.

At the same time, LDPC code check matrices $H$ with identical dimensions and number of ones can generate codes with different code distance $d$ and correcting ability $t$. Thereby, there is a problem of finding the best LDPC code check matrix with specific parameters set $n, W_{\mathrm{r}}, W_{\mathrm{c}}$ by criterion of the maximum correcting ability $t_{\max } \leq\left(d_{\max }-2\right) / 2$.

The LDPC code check matrix is presented as:

$H=\left[\frac{\frac{H_{1}}{\pi_{1}\left(H_{1}\right)}}{\frac{\ldots}{\pi_{W_{C}-1}\left(H_{1}\right)}}\right]$,

where $H_{1}$ is a basic submatrix, $\pi_{i}\left(H_{1}\right)$ are submatrices given by casual columns permutation of basic submatrix $H_{1}, i=1,2, \ldots, W_{\mathrm{c}}-1$.

LDPC code check matrix $N$ can be led to the view (3) by method of Gaussian exclusion:

$H=\left[A \mid I_{n-k}\right]$,

where $A$ is fixed matrix with dimensions $((n-k) \times k)$ that include zeros and ones and is not sparse; matrix $I_{n-k}-$ identity matrix with dimensions $(n-k) \times(n-k)$.

The matrix $G$ for generating the code words:

$G=\left[I_{k} \mid-A^{T}\right]$,

where $I_{k}$ - identity matrix with dimensions $k \times k ; A^{T}$ - transposed matrix from a matrix $A$.

If the check matrix $H$ is presented in the form (3), then generation matrix $G$ (4) can be deduced from check matrix $N$ by transformations. $G$ matrix also is called as generating matrix as far as code words are all possible linear combinations of rows in matrix $G$. Matrices $N$ and $G$ are connected by expression (MacWilliams and Sloane, 1977):

$G H^{T}=0, H G^{T}=0$.

Forming of a code word is performed based on multiplication and addition operations on information bits and lines of generation matrix $G$. Decoding a code word can be performed by numerous different iterative methods.

The task consists in search of the analytical dependence for LDPC code correcting ability $t$ as a function of the code word length $n$ and the code rate $R$ based on carrying out multiple numerical experiments with analysis the LDPC code check matrices $H$.

Input information: randomly generated check matrices $H$ with parameters: $n, W_{\mathrm{r}}, W_{\mathrm{c}}$.

Intermediate information: correcting ability $t$ of each check matrix $H$. 
Output information: the experimental and analytical dependencies of maximal LDPC code correcting ability $t$ as a function of the code word length $n$ and the code rate $R$ :

$t=f(n, R)$.

\section{Research of the LPDC Code Analytical Description}

Thousands of random check matrices $H$ are generated with specific parameters sets: $n, W_{\mathrm{r}}, W_{\mathrm{c}}$. The best characteristics of generated LDPC code check matrices are presented in the coordinates (6), where $d$ is LDPC code distance: $d=2 t+2$ (Fig. 1). The best characteristic means a maximum number of corrected errors $t$ in a code word with length $n$ bits. In this work, the check matrices for code word length $n$ are examined: 50, 100, 200, 500, 1000 bits. The gray solid line on Figure 1 designates the Plotkin limit that shows the border for existence of block codes in coordinates (6) (MacWilliams and Sloane, 1977). Anti-noise block code does not exist above the Plotkin limit, and if code is closer to this limit, then code is better by criteria of high code rate value and the maximum number of corrected bit errors per a code word. The Plotkin limit on Figure 1 is applicable for code word length with hundreds and thousands bits per a code word.

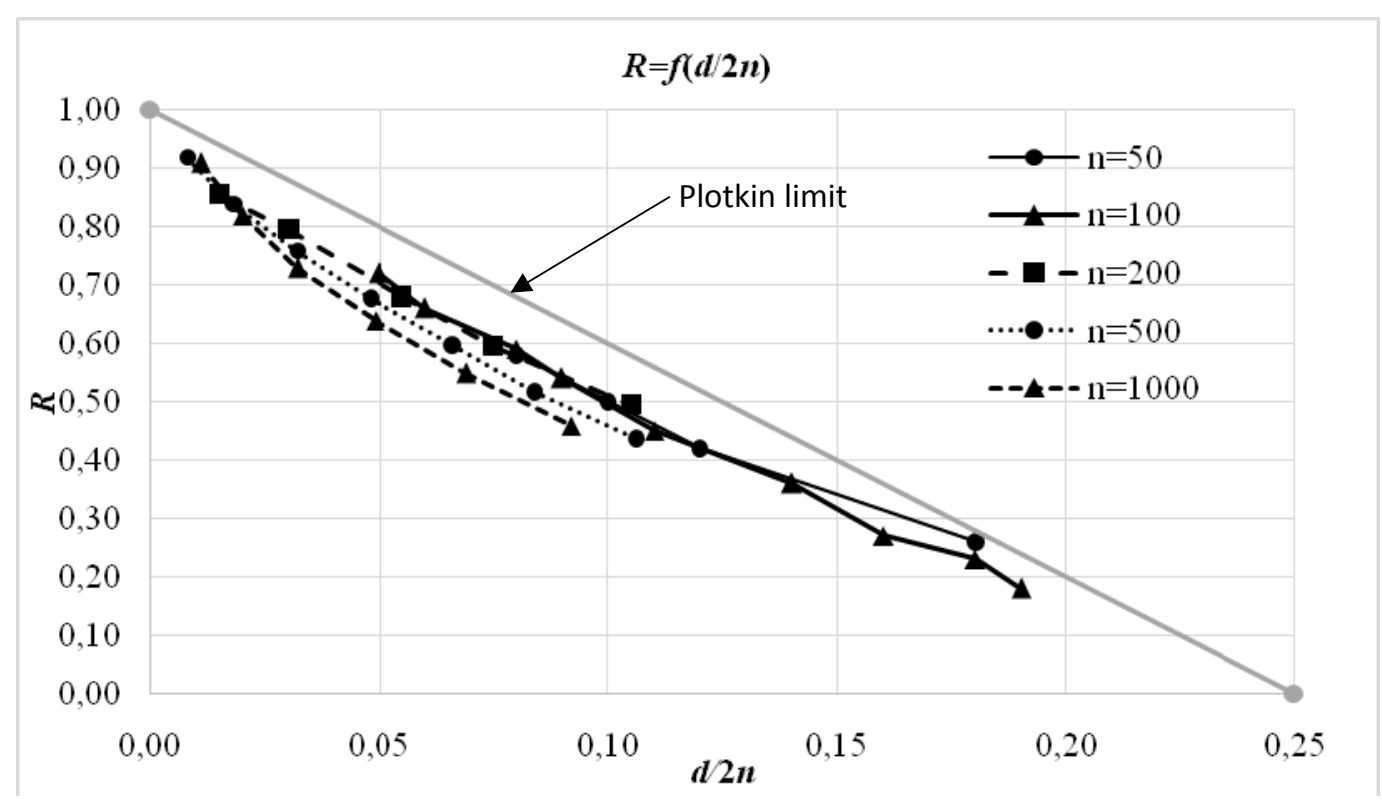

Figure 1. Experimentally given dependencies for LDPC codes: $R=f\left(\frac{d}{2 n}\right)$

As it is shown above, the code distance for LDPC code is $d=2 t+2$. If divide both parts on $2 n$, then we receive: $\frac{d}{2 n}=\frac{2 t+2}{2 n}=\frac{t+1}{n}$, from where $R=f((t+1) / n)$ and $t=f(n, R)$. The dependency $t=f(n, R)$ for regular LDPC codes with code word length $n=50 \ldots 1000$ bits is studied below.

\subsection{Linear approximation}

It is possible to match a linear approximation for every experimental line on the Figure $1 R=f(d / 2 n)$ for different $n$ values: $R=f((t+1) / n) ; y=k_{1} x+k_{2}$, where $k_{1}, k_{2}-$ factors of linear function, $x=(t+1) / n-$ argument. The factors of linear approximation are presented in Table 1 for every studied code length value $n$. 
Table 1. Factors of linear approximation

\begin{tabular}{|c|c|c|}
\hline $\mathbf{n}$ & $\mathbf{k}_{\mathbf{1}}$ & $\mathbf{k}_{\mathbf{2}}$ \\
\hline 50 & -3.1429 & 0.8171 \\
\hline 100 & -3.7582 & 0.8871 \\
\hline 200 & -4.0713 & 0.912 \\
\hline 500 & -4.8146 & 0.927 \\
\hline 1000 & -5.3911 & 0.9293 \\
\hline
\end{tabular}

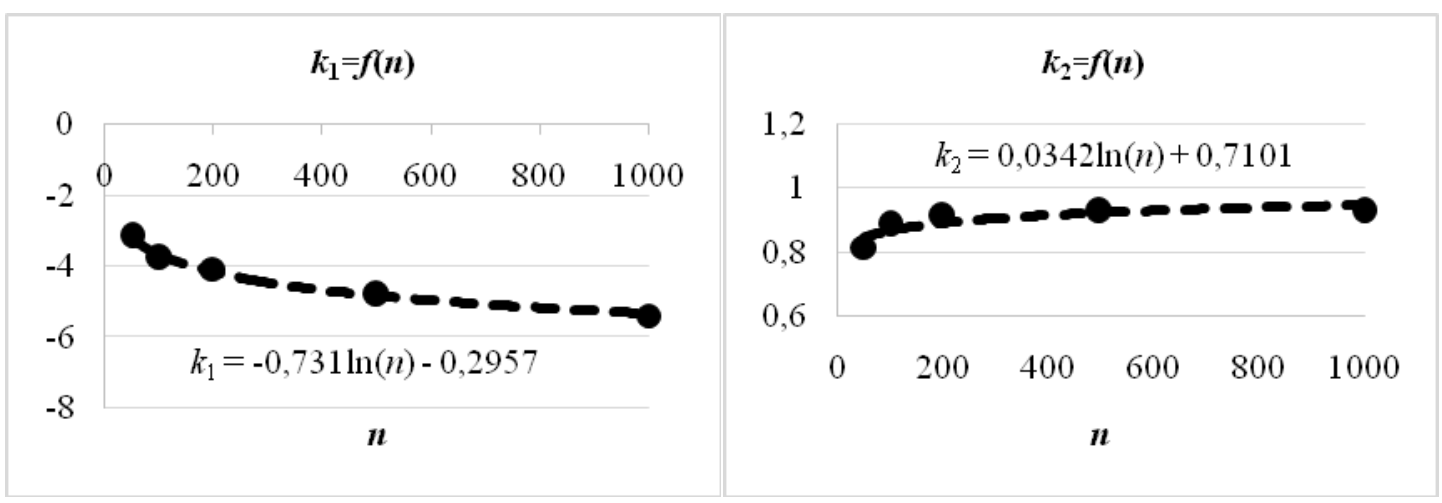

$\mathrm{a}$

b

Figure 2. Approximation of factors $k_{1}$ (a) and $k_{2}$ (b) for linear function $R=k_{1} \cdot \frac{t+1}{n}+k_{2}$

Thus,

$R=f\left(\frac{t+1}{n}\right)=k_{1} \cdot \frac{t+1}{n}+k_{2}$,

$t=\frac{n\left(R-k_{2}\right)}{k_{1}}-1$.

As it is shown on Figure 2,

$k_{1}=-0,731 \ln n-0,2957$,

$k_{2}=0,0342 \ln n+0,7101$.

The expression for linear approximation $t=f(n, R)$ becomes as:

$t=\left\lfloor\frac{n(R-0,0342 \ln n-0,7101)}{-0,731 \ln n-0,2957}-1\right\rfloor$.

\subsection{Exponential approximation}

Similarly, every line presented on Figure $1 R=f(d / 2 n)$ for different code word lengths $n$ can be described by exponential approximation $R=f((t+1) / n): y=k_{1} e^{k_{2} x}$, where $k_{1}, k_{2}-$ factors of exponential approximation, $x=(t+1) / n$ - argument. The factors of exponential approximation are presented in Table 2 for every studied code length value $n$. 
Table 2. Factors of functions of exponential approximation

\begin{tabular}{|c|c|c|}
\hline $\mathbf{n}$ & $\mathbf{k}_{\mathbf{1}}$ & $\mathbf{k}_{\mathbf{2}}$ \\
\hline 50 & 1.1106 & -8.066 \\
\hline 100 & 1.3427 & -10.05 \\
\hline 200 & 0.9831 & -6.583 \\
\hline 500 & 0.9842 & -7.647 \\
\hline 1000 & 0.9676 & -8.174 \\
\hline
\end{tabular}

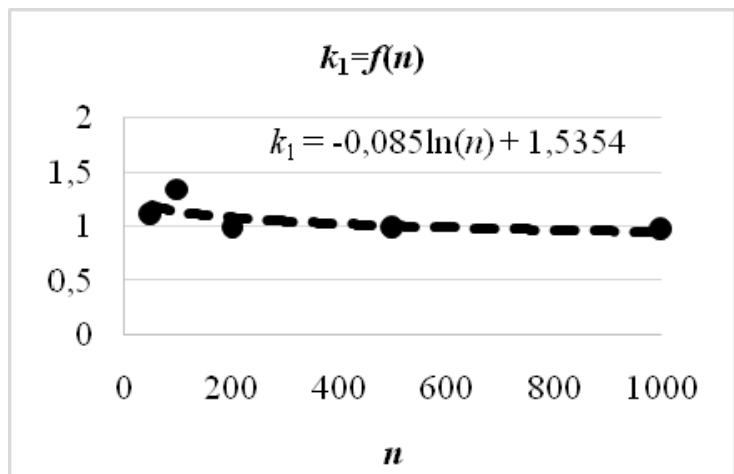

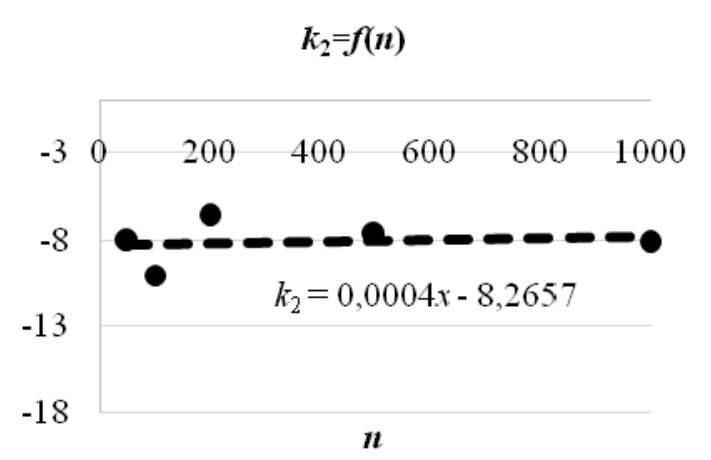

$\mathrm{b}$

Figure 3. Approximation of factors $k_{1}(\mathrm{a})$ and $k_{2}(\mathrm{~b})$ for exponential function $R=k_{1} \exp \left(k_{2} \frac{t+1}{n}\right)$

Thus,

$R=f\left(\frac{t+1}{n}\right)=k_{1} e^{k_{2} \frac{t+1}{n}}$,

$t=\frac{n}{k_{2}} \ln \frac{R}{k_{1}}-1$.

As it is shown on Figure 3,

$k_{1}=-0,085 \ln n+1,5354$,

$k_{2}=0,0004 n-8,2657$.

The expression for exponential approximation $t=f(n, R)$ becomes as:

$t=\left\lfloor\frac{n}{0,0004 n-8,2657} \ln \frac{R}{1,5354-0,085 \ln n}-1\right\rfloor$.

\subsection{Polynomial approximation}

Every line $R=f(d / 2 n)$ that is presented on Figure 1 for different code word lengths $n$ can be described by polynomial approximation $R=f((t+1) / n): \quad y=k_{1} x^{2}+k_{2} x+k_{3}$, where $k_{1}, k_{2}, k_{3}-$ factors of function, $x=(t+1) / n-$ argument. The factors of polynomial approximation are presented in the Table 3 for every studied code length value $n$. 
Table 3. Factors of functions of polynomial approximation

\begin{tabular}{|c|c|c|c|}
\hline $\mathbf{n}$ & $\mathbf{k}_{\mathbf{1}}$ & $\mathbf{k}_{\mathbf{2}}$ & $\mathbf{k}_{\mathbf{3}}$ \\
\hline 50 & 12.707 & -6.5193 & 1.0215 \\
\hline 100 & 13.199 & -7.2674 & 1.0983 \\
\hline 200 & 14.746 & -6.1859 & 0.98 \\
\hline 500 & 21.377 & -7.4009 & 0.9861 \\
\hline 1000 & 36.906 & -9.1433 & 0.9948 \\
\hline
\end{tabular}

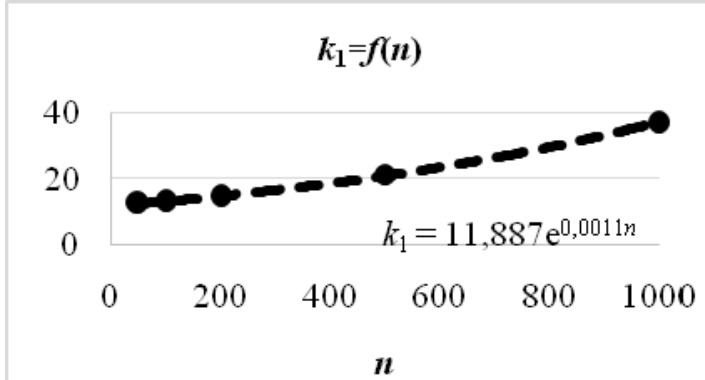

a

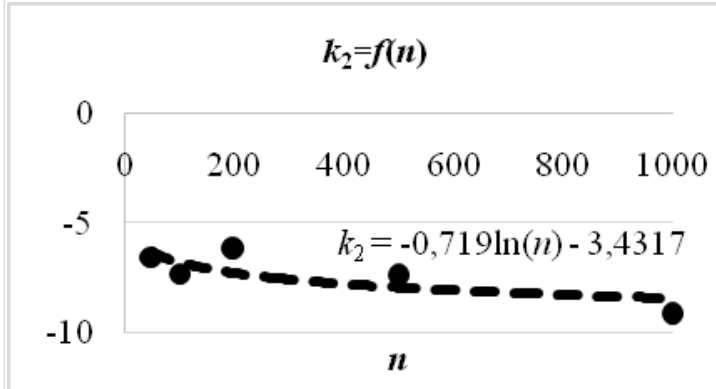

b

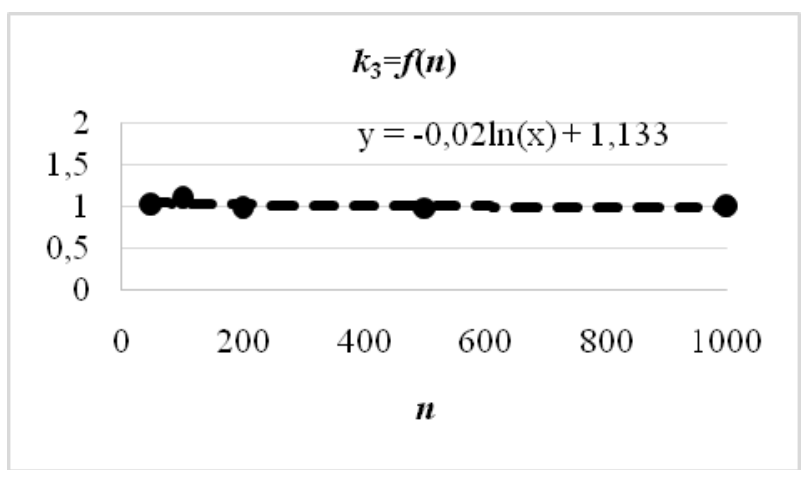

C

Figure 4. Approximation of factors $k_{1}(\mathrm{a}), k_{2}(\mathrm{~b})$ and $k_{3}(\mathrm{c})$ for polynomial function $R=k_{1}\left(\frac{t+1}{n}\right)^{2}+k_{2}\left(\frac{t+1}{n}\right)+k_{3}$

Thus,

$R=k_{1}\left(\frac{t+1}{n}\right)^{2}+k_{2}\left(\frac{t+1}{n}\right)+k_{3}$

$t=\left\lfloor\frac{-\left(2 k_{1}+n k_{2}\right)-\sqrt{\left(2 k_{1}+n k_{2}\right)^{2}-4 k_{1}\left(k_{1}+n k_{2}+n^{2} k_{3}-n^{2} R\right)}}{2 k_{1}}\right\rfloor$,

where

$k_{1}=11,887 e^{0,0011 n} ;$

$k_{2}=-0,719 \ln n-3,4317$;

$k_{3}=-0,022 \ln n+1,1336$. 
The comparison of LDPC code correcting ability values $t$ that is calculated based on the deduced analytical expressions (linear (9), exponential (12) and polynomial (14)) and experimentally given values of LDPC code correcting ability values $t$ (Fig. 1) based on simulation modelling and check matrices analysis, shows the following results:

- The analytical expression $t=f(n, R)$ (12) for exponential approximation the most precisely describes the dependence $t=f(n, R)$ from the three deduced approximations (9), (12), (14). The deviation from experimental values of LDPC code correcting ability stands in bounds $\Delta t_{\mathrm{dev}}=\left|t_{\text {experim }}-t_{\text {approx }}\right|=0 \ldots 2$ bits, and just in small number of point it reaches $\Delta t_{\text {dev }}=3$ bits;

- The analytical expression $t=f(n, R)$ (14) for polynomial approximation also enough precisely describes the dependence $t=f(n, R)$ and $\Delta t_{\text {dev }}=\left|t_{\text {experim }}-t_{\text {approx }}\right|=0 \ldots 3$ bits, but in separate individual points the deviation of an analytically given value reaches $\Delta t_{\mathrm{dev}}=3 \ldots 6$ bits;

- The analytical expression $t=f(n, R)$ (9) for linear approximation the least precisely matches with experimental results by criterion of compliance the calculated values with expression (9) and experimentally given values (Fig. 1). In some cases the deviation $\Delta t_{\text {dev }}=\left|t_{\text {experim }}-t_{\text {approx }}\right|$ reaches up to 10 bits, mostly when code rate $R$ tend closely to 1 . This is an expected result because of obviously nonlinear experimental characteristic $R=f(d / 2 n)$ (Fig. 1).

The most precise analytical expression $t=f(n, R)(12)$ is plotted on Figure 5 for code lengths 200,500 and 1000 bits.

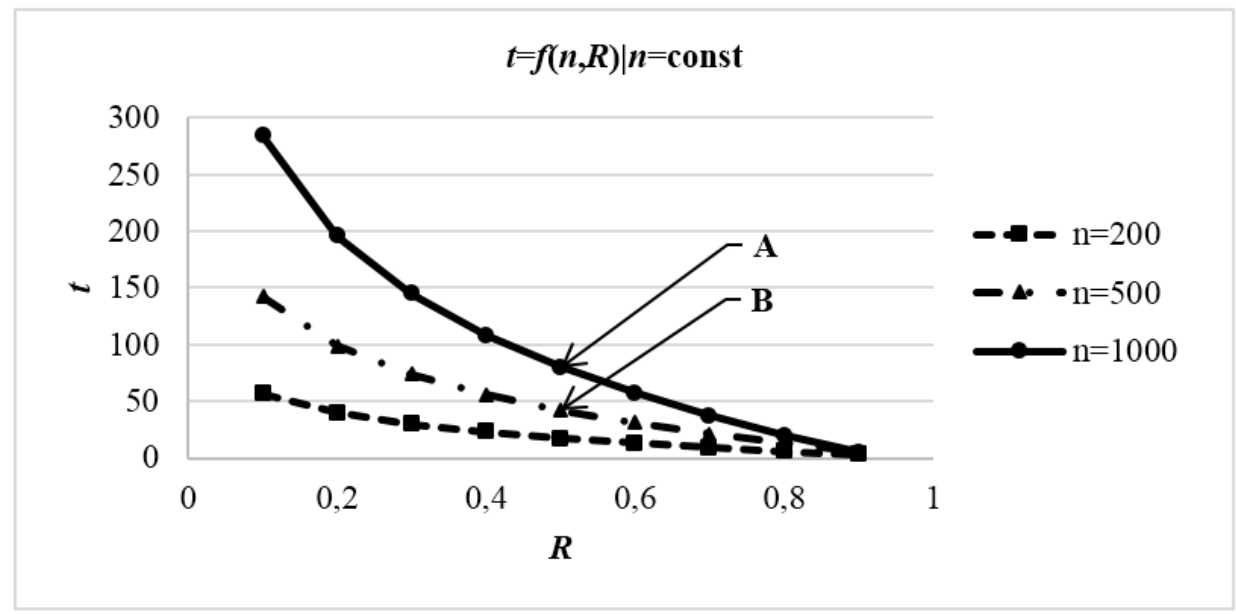

Figure 5. The exponential approximation $t=f(n, R)$ (12) for code lengths 200,500 and 1000 bits

Two points are pointed out on Figure 5: A $(n=1000 ; R=0,5 ; t=80)$ and $\mathrm{B}(n=500 ; R=0,5 ; t=42)$. These points show the exact number of maximal number of corrected errors $t$ bits per a LDPC code word with length $n$ bits and code rate $R$. The paper (Uryvsky, Prokopenko and Pieshkin, 2011) proposes the method for search the best anti-noise code to meet the required BER on the receiver side and keep maximal information efficiency. Following this method, if the next parameters are known: signal to noise rate in the receiver point, modulation technique, required BER on the receiver side, then the deduced formula (12) for LDPC code can be used for calculation the LDPC code parameters $(n, R, t)$ that is able to correct specific number of errors and keep a maximal information efficiency. 


\section{Conclusion}

Regular LDPC codes error correcting ability per a code word is explored based on the analysis of multiple randomly generated check matrices of LDPC code with code word length $50 \ldots 1000$ bits and code rates $0,15 \ldots 0,92$. The analytical expressions are investigated for regular LDPC code error correcting ability in this work based on the results given experimentally for LDPC codes error correcting ability $t$, bits.

Three analytical expressions for approximation the regular LDPC code error correcting ability are given: linear, exponential and polynomial.

The exponential approximation (12) is recommended as the most exact analytical expression for definition the LDPC codes error correcting ability following the results of comparison the experimental and analytically calculated number of bits that are corrected per a code word. The deviation of analytically calculated values and experimentally given values stands in bounds $0 \ldots 2$ bits for the exponential approximation, and only in rare cases, the deviation can be 3 bits.

The investigated exponential approximation can be used for a definition the exact LDPC code that is able to correct a specific set of bit errors per a code word, meet the required bit error rate on a receiver side, and reach maximal information efficiency.

\section{References}

1. Gallager, R. (1963) Low-Density Parity-Check Codes. Cambridge: MIT Press.

2. Uryvsky, L., Prokopenko, K., Pieshkin, A. (2011) Noise combating codes with maximal approximation to the Shannon limit. Telecommunication Sciences, 2(1), 41-46.

3. MacKay, D.J.C., Neal, R.M. (1996) Near Shannon limit performance of low-density parity check codes. IEE Electronics Letters, 32(18), 1645-1646.

4. Luby, M., Mitzenmacher, M., Shokrollahi, A., Spielman, D. (1998) Improved low-density paritycheck codes using irregular graphs and belief propagation. SRC Technical Note, $9 \mathrm{p}$.

5. Ping. L., Leung, W.K., Phamdo, N. (1999) Low-density parity-check codes with semi-random paritycheck matrix. IEE Electronics Letters, 35, 38-39.

6. Honary, B., Kou, Y., Xu, J., Lin, S. (2004) Construction of low-density parity-check codes based on balanced incomplete block designs. IEEE Trans. Inf. Theory, 50(6), 1257-1269.

7. Ohtsuki, T. (2007) LDPC codes in communications and broadcasting. IEIC Trans. Commun., $90-\mathrm{B}(3), 440-453$.

8. Osypchuk, S. (2014) The analytical description of regular LDPC codes error-correcting ability. In Proceedings of the $12^{\text {th }}$ International Conference "Modern Problems of Radio Engineering, Telecommunications and Computer Science", p. 76. Lviv-Slavske, Ukraine.

9. MacWilliams, F.J., Sloane, N.J.A. (1977) The Theory of Error-Correcting Codes. NJ: Bell Laboratories, USA. 